\section{El tiempo en el cuidador del paciente con cáncer, un abordaje cualitativo}

ISSN-PRINT

$1794-9831$

E-ISSN 2322-7028

Vol. 15 No. 1

Ene - Jun 2018

Cúcuta, Colombia

\section{The time of the caregiver of a patient with cancer, a qualitative approach}

\section{O tempo no cuidador do paciente com câncer, uma abordagem qualitativa}

\author{
Andrés Camargo Sánchez* \\ Helia Lised Pachón Rodriguez** \\ Diana Paola Gutiérrez Díaz de Azevedo*** \\ Wilson Andrés Parra Chico ${ }^{* * * * *}$ \\ Carmen Lucia Niño Cardozo ${ }^{* * * * *}$
}

\section{Resumen}

El tiempo, como magnitud física en su concepción más clásica, es para el ser humano una construcción y una representación mental subjetiva que moldea la percepción del mismo en relación a su velocidad. De ahí que es susceptible de variación de acuerdo a una multiplicidad de conceptos, incluyendo creencias, el propio estado de ánimo y la percepción de salud física y mental. Igualmente, el pasado, el futuro y el presente son representaciones subjetivas que dependen de factores como la percepción de tiempo, la cultura y el tiempo biológico de cada persona. Basado en lo anterior, el objetivo del presente artículo fue reflexionar sobre el concepto de tiempo y su importancia para el cuidador del paciente con cáncer, durante las fases de la enfermedad. Para lograr dicho objetivo se planteó la realización de una investigación de tipo cualitativo a través de la narrativa, el relato biográfico y la lectura intertextual, apoyándose del libro "Los Sueños de Einstein". Se encontró que no existe una única forma de definir el tiempo como una medida rígida, lineal y secuencial, sino que cada persona define y vive su propio concepto de tiempo a través de su propia experiencia e historia temporal, pudiendo ser un determinante, los procesos de salud-enfermedad. Se concluye, entonces, que la percepción del tiempo se construye por medio de la interacción del tiempo externo, los sucesos a lo largo de la vida y el tiempo interno que permite al ser humano adaptarse y afrontar los desafíos cotidianos

Palabras Clave: Tiempo, Cuidadores, Enfermedad, Percepción.

\section{Abstract}

The time as a physical magnitude in its most classical conception, is for the human being, a construction and a subjective mental representation that molds its perception related to its velocity. From there it is susceptible to variation according to the multiplicity of concepts, including beliefs, the state of mind and the perception of physical and mental health. Similarly, the past, the future and the present are subjective representations that depend on factors such as time perception, culture and biological time of each person. Based on this, the objective of this article was to reflect on the concept of time and its importance for the caregiver of a patient diagnosed with cancer, during the phases of the disease. To accomplish this objective a qualitative research was performed though the narrative, the bibliographical story, and the intertextual reading, relying on the book "The Dreams of

\section{Para citar este artículo/ To reference this article / Para citar este artigo/}

Camargo Sánchez A, Pachón Rodríguez HL, Gutiérrez Díaz de Azevedo DP, Parra Chico WA, Niño Cardozo CL. Intervención de enfermería en la administración de medicamentos en pediatría. Rev. cienc. cuidad. 2018;15(1):123-134

Este es un artículo bajo la licencia CC BY (https://creativecommons.org/licenses/by/4.0/) @ @
Recibido:

7 Abril

2017

Aprobado

27 Septiembre 2017

Autor de correspondencia

$\triangle$ Enfermero. MaCandidato a Doctor en Ciencias de la Salud. Correo: andcamargo@udca.edu. co. Orcid: 0000-0003Asociado. Universidad de Ciencias Aplicadas y Ambientales.

U.D.C.A. Bogotá,

Colombia

Enfermera. Ejecutora PAI AIEPI-TB-

LEPRA. Alcaldía

Municipal de Tausa.

Tausa, Colombia.

Correo electrónico:

Yiye2408@ hotmail.

com

*** Enfermera. Magister en Educación, Estudiante de Doctorado en Cognición y Lenguaje. Correo: diana. gutierrez0922@gmail. com. Orcid: 00000003-2865-7824. Universidade Estadual do Norte Fluminense Darcy Ribeiro. Rio de Janeiro, Brasil.

**** Medico. Magister en Fisiología. ProfeSabana. Chía, Colombia. Correo: waparrac@unal.edu.co

****** Enfermera. Magister en Bioética. Profesora Asistente. Javeriana. Bogotá, 0000-0002-1088 7501. Correo: ninocarmen@javeriana. edu.co gister en Enfermería, 4723-9310. Profesor sor. Universidad de la Pontificia Universidad Colombia. Orcid: 
ISSN-PRINT

1794-9831

E-ISSN 2322-7028

Vol. 15 No. 1

Ene - Jun 2018

Cúcuta, Colombia

Einstein". It was found that a unique form to measure time as a rigid, lineal, and sequential measure does not exist, but that each person defines and lives its own concept of time throughout their own experiences and temporal history, with the processes of health-disease being a possible determinant. It is concluded, then, that the perception of time is constructed through the interaction of external time, the events throughout life and the internal time that allows the human being to adapt and face the daily challenges.

Keywords: Time, Caregivers, Disease, Perception

\section{Resumo}

O tempo, como magnitude física em sua concepção mais clássica, é para o ser humano uma construção e uma representação mental subjetiva que molda a percepção do mesmo em relação a sua velocidade. De aí que é susceptível de variação de acordo a uma multiplicidade de conceitos, incluindo crenças, o próprio estado de ânimo e a percepção de saúde física e mental. Igualmente, o passado, o futuro e o presente são representações subjetivas que dependem de fatores como a percepção do tempo, a cultura e o tempo biológico de cada pessoa. Baseado no anterior, o objetivo do presente artigo foi refletir sobre o conceito de tempo e sua importância para o cuidador do paciente com câncer, durante as fases da doença. Para alcançar dito objetivo se planteou a realização de uma pesquisa de tipo qualitativo através da narrativa, o relato biográfico e a leitura intertextual, apoiando-se do livro "Os Sonhos de Einstein". Encontrou-se que não existe uma única forma de definir o tempo como uma medida rígida, linear e sequencial, senão que cada pessoa define e vive seu próprio conceito de tempo através de sua própria experiência e histórico temporário, pudendo ser um determinante dos processos de saúde-doença. Conclui-se, então, que a percepção do tempo se construí por médio da interação do tempo externo, os sucessos ao longo da vida e o tempo interno que permite ao ser humano adaptar-se e enfrentar os desafios cotidianos.

Palavras-chave: Cuidadores, doença, percepção, tempo.

\section{Introducción}

Aunque en general un individuo no es consciente de ello, la percepción del tiempo guía el comportamiento. El lector puede imaginarse que está conduciendo un automóvil y llega a una luz roja. Parece estar esperando mucho tiempo para que la luz cambie a verde. ¿Cómo sabe cuándo puede asumir que la luz se ha dañado y debe conducir a través de ella? Dos cosas son indispensables para tomar esa decisión: 1) Es necesario tener algún mecanismo de sincronización, como un reloj interno, para registrar la duración de la señal que ha pasado y 2) Se necesitan algunos criterios mnemónicos contra los cuales esta entrada sensorial puede ser comparada. Esto es, sólo puede saberse que se ha estado esperando demasiado tiempo en la luz roja si se tienen experiencias anteriores respecto a las duraciones de la luz roja almacenadas en la memoria.

Un aprendiz en conducción no puede determinar cuándo ha pasado demasiado tiempo porque no ha construido un criterio temporal para la duración de luz roja de un semáforo en su memoria. Este ejemplo ilustra cómo, tanto la percepción de la duración de la señal actual como el recuerdo de duraciones anteriores, son esenciales ambas para la discriminación de duración de un estímulo en concreto (1). Siguiendo esta línea de reflexión, la percepción del tiempo depende, entonces, tanto de características internas del individuo -percepción, cognición y características neurológicas-, como de la experiencia subjetiva que tiene de éste, cómo la construye y reconstruye a lo largo de su vida, interviniendo factores importantes tales como características culturales, sociodemográficas y emocionales.

El diagnóstico y progresión del cáncer es un evento que interrumpe la realidad no sólo para la persona que lo padece sino también para quienes están relacionados con ella, especialmente los miembros de su familia. Por lo general, la responsabilidad de cuidar a estos pacientes recae en la familia, ya sea un miembro de la familia inmediata -cónyuge, hijo o hermano-, o un amigo o vecino (2).

En este sentido, el cuidado de una persona que ha sido diagnosticada con cáncer es una actividad de atención directa y continua durante las 24 horas del día por parte de la familia y de los profesionales de la salud. Esta actividad debe adecuarse a los requerimientos 
y necesidades del paciente, los cuales varían, en tiempo, en un rango de segundos, minutos, horas, días, semanas, meses y años. Bajo esta perspectiva, la relación cuidado versus tiempo es generalmente abordada bajo una visión mecanicista, al estar marcada únicamente por el reloj y el calendario.

Teniendo en cuenta lo anterior, se hace posible pensar en la posibilidad de que la percepción del tiempo en cuidadores de pacientes con cáncer pueda estar influenciada por su propia experiencia del cuidado y por la forma en la que interpretan y afrontan la enfermedad de la persona a cargo; es decir, cómo la percepción del pasado, el presente y el futuro puede ser influenciada por las actuales interpretaciones y conclusiones que tienen acerca de la salud del paciente e incluso de su propio estado de salud tanto físico como mental.

En consecuencia, el objetivo del presente artículo consiste en describir el concepto de tiempo y su importancia para el cuidador del paciente con cáncer durante todas las fases de la enfermedad, a partir de los relatos biográficos de un cuidador de paciente con cáncer. Se utilizó la narrativa clínica, el relato biográfico y la lectura intertextual, apoyándose en el libro "Los Sueños de Einstein".

Para ello, se involucró tanto el constructivismo como la fenomenología, lo que enmarcó la comprensión de las interpretaciones y significados personales atribuidos a un diagnóstico de cáncer dentro de la vivencia propia del cuidado, con el fin de guiar el análisis de la experiencia subjetiva del tiempo en los contextos de enfermedad y cuidado.

Este ejercicio reflexivo fue aprobado por el comité de ética institucional de la Universidad de Ciencias Aplicadas y Ambientales U.D.C.A., obteniéndose el respectivo consentimiento informado por parte del participante.

\section{Desarrollo}

\section{Epidemiologia del cáncer en Colombia}

Datos derivados de estudios nacionales muestran que la tasa de incidencia ajustada por edad anual para todos los cánceres, excepto cáncer de piel, para el año 2006 fue de 186,6 casos por cada 100.000 habitantes en hombres y 196,9 casos en mujeres (3), encontrándose un aumento de mortalidad general por cáncer para Colombia tanto en niños como en adultos. El aproximado para el año 2015 fue de 43.804 muertes en todas las edades, en mujeres 22.288 y en hombres 21.516 (4). En virtud de lo anterior, para Colombia el cáncer es un problema de salud pública que va en aumento y que representa importantes repercusiones psicológicas, emocionales, sociales y económicas, constituyéndose en un reto actual para el sistema de salud (3).

\section{La experiencia de la enfermedad y su impacto en la percepción del tiempo}

En general, los seres humanos utilizan un esquema de vida individual que proporciona orden y propósito a la vida y que es el resultado de un conjunto de creencias personales e interpretaciones sobre su papel y propósito en el mundo. Lo anterior se ha llamado comúnmente "significado global" (5), permaneciendo relativamente estable a lo largo de la vida, incluso en momentos de dificultades. No obstante, el diagnóstico de una enfermedad como el cáncer marca para el paciente y el cuidador una ruptura en dicho "significado global", siendo el comienzo de una nueva toma de significado asociada con la experiencia de la enfermedad, la cual sólo termina cuando la persona es capaz de incorporar los cambios derivados de la enfermedad en un nuevo esquema de vida, estableciendo y sosteniendo un nuevo sistema de tiempo, orden y propósito $(5,6)$.

La investigación sobre la experiencia de la enfermedad muestra que la gravedad y cronicidad de la misma representa para el paciente y su cuidador una amenaza no sólo para la salud física, sino también para el sentido de individualidad e identidad de la persona (7). En este orden de ideas, estudios anteriores han identificado tres hallazgos claves sobre la experiencia de la enfermedad implicados en la percepción del tiempo: 1) "Pérdida de sí mismo" asociada con la enfermedad y como variable importante del curso de la vida; 2) "Disrupción biográfica" a través de la separación temporal causada por la enfermedad, y 3) una reconstrucción narrativa como un proceso a través del tiempo para mitigar la disrupción biográfica y conducir a un sentido de recuperación o de identidad renovada (8-10).

¿Qué es el tiempo?

Una de las palabras más usadas es "tiempo". Sin embargo, muchos pueden ser los interrogantes al 
ISSN-PRINT

1794-9831

E-ISSN 2322-7028

Vol. 15 No. 1

Ene - Jun 2018

Cúcuta, Colombia respecto: ¿Qué es el tiempo?, ¿Cómo varía de acuerdo a las situaciones y el contexto en el que se está? El tiempo y su medición dependen de construcciones subjetivas que varían de acuerdo con los cambios en conceptos, creencias, necesidades de la sociedad y avances tecnológicos. De igual manera ocurre con el pasado, el futuro y el presente, que son también representaciones subjetivas que dependen del tiempo mental de cada persona, el tiempo biológico y las características culturales. No hay, entonces, una talla única para todos los tiempos, sino más bien un momento diferente y subjetivo para cada individuo (11). La construcción de la percepción y representación del tiempo está mediada por factores objetivos (fisiológicos, físicos y cognitivos) y subjetivos (culturales, sociales y psicológicos) (11).

Por otro lado, es evidente la constante preocupación entre diferentes culturas sobre el paradigmático "tiempo". Un claro ejemplo es el relato de "El abrazo de la serpiente", película colombiana que muestra la cosmovisión de los indígenas de las selvas del Amazonas acerca de conceptos de memoria, tiempo y su influencia en la historia de vida, que en este caso se aborda como un tiempo espiral (12) (Figura 1).

La evidencia científica muestra que los seres humanos de una cultura particular experimentan interiormente la actitud convencional hacia el tiempo y consecuentemente se comportan temporalmente de acuerdo con su patrón internalizado (13). Así, individuos en diferentes culturas desarrollan diferentes estrategias para usar y medir el tiempo y esas habilidades reflejan la riqueza de formas en que el tiempo subjetivo puede ser usado. Esto es relevante si se tiene en cuenta que, dentro del cuidado del paciente y el proceso de salud-enfermedad, la percepción del tiempo y su experiencia juegan un rol importante en aspectos tales como horarios de sueño, de alimentación, de medicación y de esparcimiento.

Para el presente artículo de reflexión vale la pena describir brevemente los dos tipos de tiempos conocidos. Uno es externo, construido por la interacción entre lo físico y lo cultural; el otro es interno, experimentado por todos los seres vivos, siendo mejor conocido como tiempo biológico. La sincronización y ritmicidad de estos dos tiempos es vital para la adaptación; es sinónimo de salud y bienestar en tanto permite entender el pasado, el presente y el futuro en una compleja interacción fisiológica, psicológica y comportamental.

En un sentido físico, el tiempo también es entendido como de tipo mecánico, siendo una magnitud física con la que es posible medir la duración o separación entre acontecimientos que pueden ser cíclicos y repetitivos, como son la rotación y traslación terrestre. Es a partir del tiempo, entendido como entidad mecánica, que ha sido posible para diferentes sociedades la construcción de sistemas de medida para cuantificar su paso o transcurso, influenciados por características culturales, religiosas y tecnológicas. Por ejemplo, mientras que los cristianos utilizan el calendario solar gregoriano, el islamismo y judaísmo han conservado el calendario lunar, donde la observación de la luna creciente marca el inicio de cada fiesta religiosa.

Por otro lado, se encuentra el tiempo corporal, un fenómeno que afecta la actividad biológica de los organismos desde el nivel celular hasta el sistémico, constituyéndose así lo que se conoce como ritmos biológicos -conjunto de fenómenos fisiológicos que se presentan de forma cíclica en los organismos vivos-, comúnmente clasificados en tres grandes grupos de acuerdo con la duración de su oscilación con respecto al día de 24 horas: ritmos ultradianos (cortos, de menos de 24 horas), ritmos infradianos (largos, con más de 24 horas y que pueden abarcar días, semanas, meses, una estación o incluso más tiempo) y los ritmos circadianos (tienen un periodo de aproximadamente un día).

Actualmente, se sabe que los ritmos biológicos están presentes en todos los seres vivos de la tierra, no constituyen un fenómeno casual ni una respuesta pasiva a las condiciones ambientales y son indispensables en un proceso de adaptación al ambiente, proporcionando un orden temporal interno fundamental para la supervivencia (13).

La enfermedad y los procesos de hospitalización constituyen un factor de riesgo importante para la alteración de los ritmos biológicos, tanto en el paciente como en su cuidador, incluyendo el de sueño-vigilia, siendo este último modificable por factores tales como la edad, la ansiedad, la depresión, el dolor, la administración de medicamentos y por el propio ambiente hospitalario (13). 


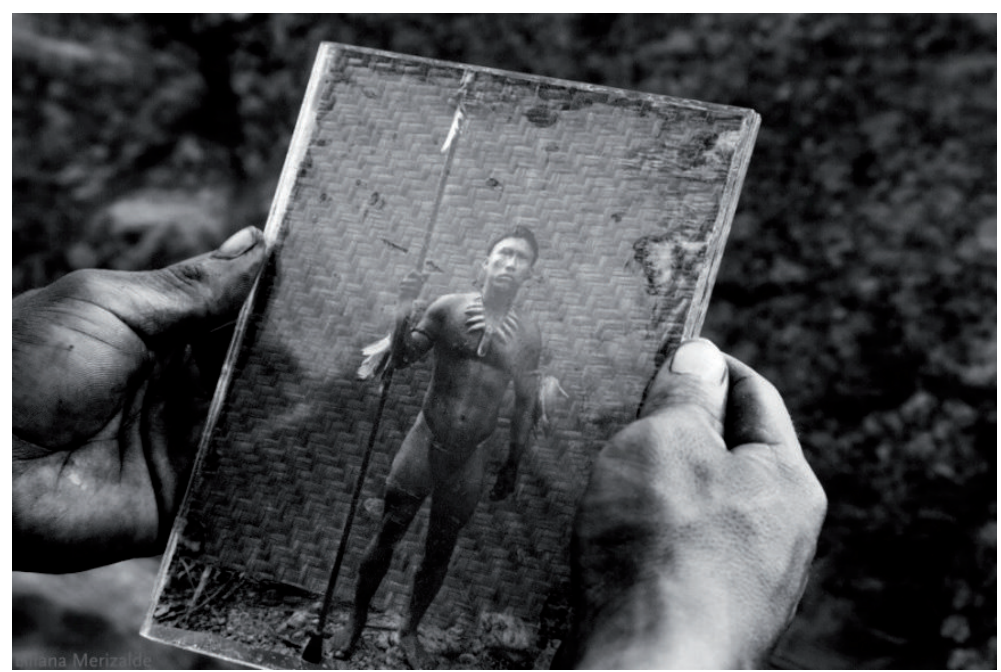

Figura 1. Chullachaqui: el cuerpo vacío o el doble vacío que deambula en el tiempo sin tiempo.

Fuente:http://www.elpais.com.co/elpais/cultura/noticias/abrazoserpiente-elegida-mejor-pelicula-festival-cine-lima

Tiempo fragmentado en pacientes con cáncer: hacia un mayor entendimiento del impacto de la enfermedad

Como resultado de un estudio previo en pacientes con cáncer, se evidencia que un elemento crítico que posiblemente emerge consistentemente en respuesta a la enfermedad es la forma en que el tiempo se fragmenta (6). Una hipótesis que da cuenta de ello plantea que es factible que el tiempo sea fragmentado por el paciente y, en algunos casos, por el cuidador debido a la carga emocional y física del cáncer, junto con la incertidumbre causada por la experiencia individual de la enfermedad. Esa restructuración del tiempo en piezas más pequeñas se hace evidente en muchas dimensiones: tiempo biográfico, tiempo de rutina, tiempo de tratamiento, días de visitas al médico, tiempo cronológico, tiempo de enfermedad, tiempo de recuperación, etc.

De esta manera, el tiempo se convierte en una abstracción para las personas con cáncer, razón por la cual los cambios entre períodos se caracterizan por la incertidumbre, considerándose entonces como un tiempo anómalo. En estos componentes del tiempo subjetivo también se ha encontrado que corresponden con períodos similares en los que los pacientes están tratando enfermedades crónicas preexistentes, ya sea a través de visitas médicas, de horarios adicionales, de control de síntomas o de llamadas telefónicas frecuentes $(6,14)$.
Para muchos pacientes con cáncer, se sabe, el tiempo antes de la enfermedad se refiere al pasado recordado (tiempo pasado), el tiempo marcado por una enfermedad crónica y el tratamiento se conoce como el presente percibido (tiempo de enfermedad) y el futuro como representación de lo que aún está por venir se asume como el futuro imaginado (tiempo futuro) (6). En este sentido, indagar sobre cómo el cáncer impacta la calidad de vida, relacionada con la salud tanto física como mental, debería incluir el estudio de la percepción del tiempo tanto en pacientes como en cuidadores, siendo claro que las dimensiones del tiempo son centrales para las experiencias individuales de la enfermedad.

\section{El presente ejercicio reflexivo}

Este artículo utiliza los relatos biográficos (escritos y orales) de un cuidador que, por razones obvias, están inundados de imaginación, emoción y asombro (15), buscando que sirvan como sustrato de reflexión y reinterpretación del concepto de tiempo.

Las narrativas son un valioso instrumento que permite la reflexión y comprensión de fenómenos -en este caso, el tiempo-, desde nuevas perspectivas, con el fin de que resulten valiosas para la investigación.

Para llevar a cabo el ejercicio narrativo, se tuvieron en cuenta tres momentos (16): 
ISSN-PRINT

1794-9831

E-ISSN 2322-7028

Vol. 15 No. 1

Ene - Jun 2018

Cúcuta, Colombia a) Atención o escucha activa, en donde se realizó una entrevista al cuidador buscando -dentro de su discursolas posibles relaciones con el concepto de tiempo.

b) Representación, en la cual, a partir de la imaginación narrativa (15) y el uso de la técnica de lectura intertextual, se propuso una reinterpretación del concepto de tiempo. En este caso, el cuidador está situado entre las experiencias del entrevistador y su intento por entenderlas y describirlas, pretendiendo que el diálogo entre los relatos permita llegar a un momento de acción narrativa.

c) Acción narrativa, que se propuso fuera realizada a manera de reflexión, lo cual implica no solamente la explicación de los hechos pasados sino también una construcción no sistemática de conexión cognitiva entre los textos. Se propuso, adicionalmente, que se pudieran generar cambios en la forma de enseñar, vivir e investigar el tiempo en los procesos de salud y enfermedad, entendiendo que hay un vínculo vital entre narrativa y acción humana.

Como se mencionó al principio, esta narrativa se apoyó con otro relato: "Los Sueños de Einstein", una intertextualidad explicitada que le confiere identidad específica al discurso mediante un conjunto de treinta distintos cuentos, cada uno de ellos describiendo un sueño ficticio del joven Albert sobre el concepto del tiempo, con una visión variada: tiempos que se detienen y vuelven a arrancar, tiempos desenfrenados, tiempos dramáticamente lentos, pasados que se confunden con presentes y múltiples futuros, todos ellos entrelazados para explorar la experiencia de cómo se percibe el tiempo, siendo uno de los conceptos más desafiantes y elusivos en el pensamiento humano (17).

De esta manera, en diferentes épocas históricas, el personaje central, que es el tiempo, protagoniza diversas situaciones para explicar sus múltiples inferencias en las experiencias de la vida, dejando ver su importancia más allá de la limitación de acontecimientos e incluyendo tiempos necesarios para vivir y cuidar de la salud humana, objetivo central de reflexión del presente artículo.

La intertextualidad que enmarca este texto responde a una multiplicidad de lecturas en una red dialógica de discurso a discurso, elemento clave para problematizar la lectura entre textos, orientando su interpretación y proponiendo espacios inter-discursivos que se intercalan entre el tiempo y el espacio. Por los tanto, el dialogismo, como condición de existencia en todo discurso, caracteriza la narrativa con la heterogeneidad propia de toda práctica social (1820). Así, los relatos de Juan (el cuidador entrevistado) y del libro "Los Sueños de Einstein" exponen un abanico de experiencias sutiles de comprensión para el lector, que posiciona también su discurso frente a la temática expuesta (21).

\section{El tiempo desde la experiencia de un cuidador principal de un paciente con cáncer y desde "Los sueños de Einstein"}

\section{4 de abril de 1905.}

"En este mundo hay dos tiempos. Un tiempo mecánico y un tiempo corporal. El primero es tan rígido y metálico como un pesado péndulo de hierro que va y vuelve, va y vuelve, va y vuelve. El segundo gira y se ondula como un pez azul en una bahía. El primero es inflexible y predeterminado. El segundo elige el futuro a medida que transcurre".

"Cuando ambos tiempos se encuentran, desesperación. Cuando ambos tiempos siguen caminos separados, alegría. Porque, milagrosamente, un abogado, una enfermera, un panadero, pueden crear un mundo en cualquiera de ambos tiempos, pero no en los dos. Cada uno es verdad, pero esas dos verdades no son la misma".

Ahora, el tiempo empieza a girar en torno a una enfermedad, en este caso el cáncer y el cuidado del paciente, ajustándose a una serie de sucesos definidos por la rutina de la enfermedad y su progresión. Las fechas son definidas por citas médicas, los días por hospitalizaciones y las horas por horarios de visita o de medicamentos. Ahora que se unen estos dos tiempos, se cae en la desesperación.

\section{8 de abril de 1905.}

"El tiempo es visible en todas partes. Relojes de las torres, relojes de pulsera, campanas de las iglesias dividen los años en meses, los meses en días, los días en horas, las horas en segundos: cada incremento temporal marcha tras otro en perfecta sucesión". 
Cuidador: "...Se vuelve uno esclavo del tiempo; hay cosas que se deben hacer, ya no se seguían fechas especiales como antes, sino que la enfermedad y su tratamiento marcaba los momentos. Por ejemplo: día de los exámenes de control, día de la quimioterapia, el tiempo los definía eventos relacionados y en torno a la enfermedad. Era curioso, hasta las conversaciones giraban en torno a lo que iba a pasar después de los controles médicos...".

Los cambios en la rutina de una persona y su familia después del diagnóstico de cáncer aumentan debido a que ahora se debe asistir a exámenes, controles médicos y tratamientos agresivos con efectos secundarios importantes que hacen que la cotidianidad y la dinámica familiar varíen (22). En esta experiencia, es común que se presenten sentimientos de tristeza y miedo, así como sintomatología ansiosa y depresiva (23), emergiendo también una conciencia de tiempo en torno al cuidado del paciente y a la lucha contra el cáncer, entendiéndose el tiempo como un recurso limitado y valioso.

\section{6 de abril de 1905.}

"En este mundo, el tiempo es como un curso de agua, ocasionalmente desplazado por un obstáculo o una brisa pasajera".

Cuidador: "El tiempo va muy relacionado en cómo se vive la vida y, ante un diagnóstico de una enfermedad catastrófica, sencillamente cambió la vida. Al principio se perdió el rumbo, después que entró el médico con los resultados de los exámenes y nos dijo que mi mamá tenía cáncer, porque ya es el tiempo que da la vida frente a un diagnóstico y un tratamiento que puede funcionar o no".

El diagnóstico de cáncer se considera como un evento vital estresante, que afecta no sólo al individuo que lo recibe sino también a su entorno familiar y social más directo. Como todo suceso estresante, el cáncer no produce el mismo impacto en todos los individuos. Si bien el proceso más común consiste en que el presente percibido o el tiempo de la enfermedad se podría definir por una experiencia más personal del cáncer, ahora la actividad principal del paciente y su cuidador se convierte en la integración del cáncer en los pliegues del contexto de la vida individual, el mantenimiento general de la salud y la recuperación del cáncer a largo plazo (6); el trabajo, la familia y los planes que se tenían, se convierten en un futuro incierto (24).

\section{1 de junio de 1905.}

"En verdad, este es un mundo sin futuro. En este mundo, el tiempo es una línea que termina en el presente, tanto en la realidad como en la mente. En este mundo, nadie puede imaginar el futuro".

Cuidador: "Generalmente se asocia cáncer con muerte; el día que dieron el diagnóstico, sólo pude pensar en la muerte de mi mamá. Sin embargo, el médico nos dio esperanzas, recuerdo mucho una frase que él dijo -es cáncer, pero se puede tratar-. Aunque era esperanzador, imaginaba lo agresivo que podían ser los tratamientos, también mi mamá nos dio fortaleza cuando nos dijo que siempre que haya cosas por hacer, habrá esperanza".

El pasado y el futuro se entrelazan en el presente, en una construcción biográfica y social determinada por el estado de salud. Un cambio en el presente va a desencadenar un cambio en el futuro y en la forma como se entienden y construyen el tiempo y la vida. Las enfermedades crónicas, entre ellas el cáncer, aunque tienen diferentes variables fisiológicas, biológicas y patológicas, comparten un hilo en el tiempo. Las enfermedades crónicas graves inducen diferentes ritmos temporales y diferentes relaciones con el tiempo, que son experimentados por la persona sana o, incluso, por la persona con enfermedad aguda. La alteración de los ritmos de la vida corporal cambia las expectativas de la persona para el futuro y sus relaciones con los demás.

Los significados atribuidos a experiencias y prácticas del pasado y del presente, así como planes y fantasías futuras, adquieren un nuevo significado cuando se recibe el diagnóstico de una enfermedad crónica como el cáncer $(25,26)$. En este punto resulta muy importante el apoyo del profesional de la salud, pues él puede favorecer la continuidad de ese hilo temporal de pasado-presente-futuro, generando expectativas, esperanza y calidad de vida a partir de la resolución de interrogantes respecto al diagnóstico, tratamiento, 
ISSN-PRINT

1794-9831

E-ISSN 2322-7028

Vol. 15 No. 1

Ene - Jun 2018

Cúcuta, Colombia pronóstico y la recidiva de la enfermedad, lo cual permite mitigar la desesperanza ante la percepción negativa del cáncer (27).

El trabajo de este profesional permitiría al paciente, su cuidador y su familia el favorecimiento de estrategias de afrontamiento eficaces, en donde el apoyo social es un factor que mitiga el impacto de los acontecimientos vitales traumáticos (27).

\section{3 de junio de 1905}

"Imagina un mundo en que la gente vive sólo un día... En este mundo en que una vida humana sólo abarca un día, la gente acecha el tiempo, como los gatos tratan de oír un sonido en el desván. Porque no hay tiempo que perder".

"El tiempo es demasiado precioso. Una vida es un momento de una estación. Una vida es una sola nevada. Una vida es un día de otoño. Una vida es el canto delicado y fugaz de la sombra de una puerta que se cierra. Una vida es un breve movimiento de los brazos y las piernas".

Cuidador: "Le di un nuevo significado al tiempo. Tenía dos caminos: perder el tiempo cuestionándome por qué nos pasó esto o tratar de aprovecharlo al máximo; le di un nuevo significado al tiempo, no sólo el que quedaba con ella, sino el que me queda a mí, de vida. Ahora debía hacer las cosas más de prisa, porque el tiempo se acaba".

"Después del diagnóstico, traté de no volver a discutir con mi familia por cosas triviales; cuando por compromisos de mi trabajo debía quedarme en la oficina, llamaba más seguido a mi mamá y los fines de semana salíamos a caminar. Creo que eso nos unió y nos dio fuerza para seguir afrontando la enfermedad y aprender que mañana puede ser tarde".

Autores como Pinquart, Frohlich y Silbereisen (28) identifican que, al enfrentar condiciones de enfermedad como el cáncer, surgen actitudes y pensamientos negativos, pero también positivos. La vida toma un giro dramático cuando se atribuye a la enfermedad el poder de provocar un cambio significativo en la vida y se hace necesario el desarrollo de actitudes positivas hacia dicha enfermedad, entre ellas un mejor uso del tiempo.

\section{5 de junio de 1905.}

"En un mundo en que el tiempo es un sentido, como la vista o el tacto, una secuencia de episodios puede ser lenta o rápida, apagada o intensa, salada o dulce, causal o sin causa, ordenada o aleatoria, según la historia anterior del observador".

Cuidador: "El tiempo, después del diagnóstico de cáncer, lo vi diferente, se hacía eterno esperando los resultados de las biopsias, de las TAC; eran semanas eternas, sentía que me iba a enloquecer".

"Los días en que le administraban la quimioterapia también trascurrían muy despacio. Era frustrante verla conectada a una máquina todo el día, yo miraba muy seguido el reloj y el tiempo no pasaba".

"Otra situación curiosa que no sé cómo explicar es que, entre los ciclos de quimioterapia, el tiempo volaba; en un abrir y cerrar de ojos ya habían pasado 21 días y se volvía a la misma tortura".

A través de la historia de la humanidad se ha cuestionado sobre la dicotomía existente entre el tiempo físico y objetivo y el tiempo subjetivo o biológico. El tiempo percibido por el ser humano sería la experiencia consciente de los procesos que le permiten adaptarse a las relaciones secuenciales de su ambiente, con lo cual las representaciones temporales serían funciones cognitivas de alto nivel.

En la última década se ha experimentado un resurgimiento en el interés hacia la percepción y la estimación de la duración del tiempo, debido a que la capacidad de estimar el paso del tiempo es de fundamental importancia para los procesos perceptivos y cognitivos (29). La experiencia del tiempo es la percepción de la duración, que no es isométrica a la duración física y puede ser distorsionada por una serie de factores externos e internos a nuestro organismo. Así, el tiempo parece transcurrir más rápido cuando se está feliz y motivado. Por el contrario, cuando se está triste y aburrido, el tiempo se percibe como más largo.

Lo anterior está a su vez influenciado por la rutina conformada por las experiencias previas. Así, una 
persona, ante una situación difícil en el curso de una enfermedad, tiene la sensación de que el tiempo subjetivo es mayor que el tiempo cronológico, lo que se constituye en un indicador de malestar (30). Generalmente, cuando hay sufrimiento, el tiempo se hace más largo (31), situación que no sucede sólo en el paciente con cáncer, sino que también está presente en la familia y sus cuidadores

Siguiendo lo anterior, la subestimación o sobreestimación (contracción y dilatación) del tiempo puede tener relevancia en la salud general y el estado físico global, así como en las actitudes y experiencias de la vivencia actual, regulando la vida diaria y cotidiana con la conocida injerencia en la adaptación al contexto (32).

\section{0 de mayo de 1905.}

"Hipotéticamente, el tiempo puede ser liso o áspero, sedoso o espinoso, duro o blando. Pero en este mundo, ocurre que la textura del tiempo es pegajosa. Partes de la ciudad se quedan adheridas a algún momento de la historia y no se pueden desprender. Y también las personas se quedan pegadas a algún momento de sus vidas y no pueden liberarse".

"La tragedia de este mundo es que nadie es feliz, tanto si se ha quedado pegado a un momento de dolor como a un momento de alegría. La tragedia de este mundo es que todos están solos. Porque una vida en el pasado no puede compartirse con el presente. Cada persona que se ha quedado pegada al tiempo está allí clavada, y sola”.

Cuidador: "Ahora recuerdo con cariño a las enfermeras; eran muy dulces y siempre en las hospitalizaciones y las quimioterapias nos inyectaban optimismo, creo que eso ayudó para no desfallecer... Con el apoyo del médico, que siempre tomaba tiempo para decirnos como iban las cosas, de él recuerdo que siempre nos daba opciones aunque las cosas estuvieran feas y el cáncer hubiera avanzado".

Cada individuo construye una historia continua y única en el tiempo a lo largo de su vida, con un pasado que le permite, al evocarlo, verificar su propia existencia (33). Ante la enfermedad de un familiar es común que se presenten sentimientos de tristeza y conductas asociadas a síntomas de depresión. La evidencia en el campo ha mostrado la forma en que los sesgos cognitivos generan que, en momentos de dificultad, se recuerden en mayor medida momentos y palabras tristes (34). Por tanto, es previsible que lo que haga o diga el profesional de la salud acerca del cuidado de un paciente con cáncer será recordado durante toda la vida de los familiares y cuidadores.

\section{1 de mayo de 1905.}

"En este mundo, con el paso del tiempo, aumenta el orden. El orden es la ley de la naturaleza, la tendencia universal, la dirección del cosmos. Si el tiempo es una flecha, esa flecha apunta hacia el orden. El futuro es configuración, organización, unión, intensificación; el pasado es azar, confusión, desintegración, disipación”.

Cuidador: "El tiempo pasó, pasó en algunos momentos muy rápido y en otros muy lento, y ahora mi vieja no está... Sin embargo, aunque estoy triste, me siento tranquilo porque se hizo lo humanamente posible y la vida me la prestó unos años más; ahora el mejor homenaje a ella es seguir adelante y construir un futuro y dejar huella como ella lo hizo".

Es así como el tiempo, sus medidas y representaciones convergen en las ciencias biológicas, las ciencias humanas y la filosofía, conocimientos que nutren el conocimiento dentro del cuidado de la salud humana. Por ello, este escrito buscó alcanzar la reflexión del lector a través de la narrativa de una historia de vida, esperando comprendiera esta variable tan compleja y determinante en la acción de cuidar.

\section{Consideraciones Finales}

A través del presente artículo de reflexión se realizó un análisis intertextual del relato biográfico de un cuidador principal de un familiar con diagnóstico de cáncer, durante el diagnóstico, tratamiento y pronóstico de la enfermedad, usando la narrativa de experiencias de Juan, relator de la historia, y el libro de ciencia ficción "Los sueños de Einstein" (21), con el propósito de lograr una construcción de conceptos y experiencias en relación con el tiempo, a través de la subjetividad. 
ISSN-PRINT

1794-9831

E-ISSN 2322-7028

Vol. 15 No. 1

Ene - Jun 2018

Cúcuta, Colombia
La narrativa y el análisis intertextual evidenciaron que el tiempo, al menos en este caso, no puede separarse de la experiencia de la enfermedad. El pasado, el presente y el futuro existieron al mismo tiempo para el cuidador y para quien experimenta la enfermedad, y estuvieron implícitos el uno en el otro. Con esto, podría ser relevante cuestionarse acerca de si las percepciones individuales de los pacientes y cuidadores sobre el futuro afectan las decisiones relacionadas con el tratamiento y cómo se daría esta relación.

Para el cuidador entrevistado en este estudio, el cáncer fue conceptualizado como una "sentencia de muerte" que hizo que se cuestionara, simultáneamente, sobre el estado actual de su familiar enfermo y el curso del futuro y el tiempo en sí. Una característica importante que emergió de la narrativa fue la fragmentación del tiempo que el cuidador realizó durante el proceso de la enfermedad. Específicamente, y al igual que en un estudio anterior con pacientes con cáncer (6), el tiempo fue fragmentado por el cuidador -y posiblemente por el paciente- en partes más pequeñas, en términos de experiencia, más manejables para la transición a través de la enfermedad: el pasado recordado, el presente percibido y el futuro imaginado.

Siguiendo esa línea, se puede pensar que la supervivencia frente a la enfermedad y el peso del presente percibido se convierten en objeto de enfoque para cuidador y paciente, fenómeno que surge de la necesidad de satisfacer las demandas del tratamiento y manejo de la enfermedad. A partir de estas demandas de tiempo, sobrevivir al presente con la enfermedad toma un peso importante para paciente, cuidador y familia, dado que de ello dependen las esperanzas de recuperación $(35,36)$.

Así, entonces, es posible que la carga emocional de la experiencia de la enfermedad pueda ser más fácil de sobrellevar para paciente y cuidador, si se interpreta en un contexto fragmentado. Dicha reconciliación entre tiempo-enfermedad probablemente ocurra debido a que el diagnóstico de cáncer cambia significativamente la forma en que paciente y cuidador piensan acerca de sí mismos y de sus vidas $(8,6,35)$.

Relacionado con ello, un estudio con pacientes con enfermedad crónica muestra, de manera interesante, cómo en la experiencia de la enfermedad el paciente fragmenta el tiempo en fechas notables -por ejemplo, diagnósticos, el fin del tratamiento, aniversarios y ocasiones similares-, utilizándolas como marcadores para el comienzo o el final de períodos de tiempo específicos (36).

Teniendo en cuenta lo anterior, el profesional de la salud no se debe limitar a considerar la hora del medicamento, las horas de visita o el día de la cita médica, sino que debe, dentro de su formación y actuar profesional, considerar que la percepción del tiempo en los seres humanos se construye entre la interacción del tiempo externo -con todo lo que sucede a lo largo de la vida- y el tiempo interno -que permite adaptarse y responder a la demandas ambientales diarias-, lo cual, en última instancia, contribuye a construir una representación propia y única de ese tiempo, en donde el pasado, el presente y el futuro no son continuos y lineales sino que varían de acuerdo a múltiples circunstancias. Esto implica, para este profesional, no ser ajeno al tiempo del paciente ni al de la familia.

Para finalizar, la principal limitación del presente artículo fue no contar con una mayor cantidad de cuidadores de pacientes con cáncer entrevistados, en aras de tener la oportunidad de analizar mayor cantidad de relatos y contrastarlos con la evidencia disponible. Para futuros estudios en este tópico, sería de relevancia indagar sobre diferencias en la percepción de tiempo en diferentes tipos de enfermedad crónica, teniendo en cuenta factores que podrían alterar dicha percepción en los pacientes: medicación, quimioterapia, diagnósticos previos, nivel de escolaridad y características culturales específicas; y para el caso del cuidador: experiencia previa de cuidado, escolaridad e, incluso, sintomatología ansiosa y depresiva. Resulta de igual relevancia analizar, en futuros estudios, cómo las decisiones personales relacionadas con el tratamiento del cáncer y los comportamientos de salud se ven directamente afectadas por la incertidumbre temporal que supone la enfermedad.

No obstante lo anterior, el presente artículo abre el panorama dentro del contexto nacional de explorar la percepción del tiempo como un determinante del proceso salud-enfermedad, un tópico casi inexplorado en Colombia y Latinoamérica y que podría tener importantes repercusiones dentro del diseño de estrategias terapéuticas para el paciente y de mitigación de la carga de la enfermedad para el cuidador y su familia. 


\section{Agradecimientos}

Los autores agradecen al participante por compartir sus experiencias y su tiempo en cada una de las entrevistas y, además, brindar una lección de vida y, como él mismo manifestó a través de una frase del actor norteamericano Michael Landon que sintetiza la lucha de los pacientes y sus familias contra esta enfermedad: "Voy a vencer este cáncer o morir en el intento".

\section{Conflicto de Intereses}

Los autores declaran no tener ningún conflicto de intereses.

\section{Referencias Bibliográficas}

1. Meck W. Neuropharmacology of timing and time perception. Brain Res Cogn Brain Res. 1996; 3: 227-242.

2. Milne D, Mulder L, Beelen H, Schofield P, Kempen G, Aranda S. Patients' self-report and family caregivers' perception of quality of life in patients with advanced cancer: how do they compare?. Eur J Cancer Care (Engl). 2006; 15: 125-132.

3. Ospina M, Huertas JA, Montaño JI, Rivillas JC. Observatorio Nacional de Cáncer Colombia. Rev. Fac. Nac. Salud Pública. 2015; 33(2): 1-15.

4. Pardo Ramos C, Cendales Duarte R. Incidencia, mortalidad y prevalencia de cáncer en Colombia, 2007-2011. Instituto Nacional de Cancerología. [Internet]. 2015 [consultado 16 de Septiembre de 2017]; v.1. p.148. Disponible en: http://www.cancer.gov.co/files/libros/archivos/incidencial.pdf

5. Lee V. The existential plight of cancer: meaning making as a concrete approach to the intangible search for meaning. Support Care Cancer. 2008; 16(7): 779-785.

6. Hannum SM, Rubinstein RL. The meaningfulness of time; Narratives of cancer among chronically ill older adults. J Aging Stud. 2016; 36; 17:25.

7. Pierret J. The illness experience: state of knowledge and perspectives for research. Sociol Health Illn. $2003 ; 25: 4-22$.

8. Charmaz K. Loss of self: A fundamental form of suffering in the chronically ill. Sociol Health Illn. 1983; 5(2):168-195.

9. Bury M. Chronic illness as biographical disruption. Sociol Health Illn. 1982; 4(2):167-182.

10. Williams G. The genesis of chronic illness: Narrative reconstruction. Sociol Health Illn. 1984; 6(2):175-200.

11. Tordjman S. Time and its representations: At the crossroads between psychoanalysis and neuroscience. J Physiol Paris. 2011; 105(4):137-148.

12. Rojas Sotelo M. De raíz, extracciones y apropiaciones. Estudios Artísticos: revista de investigación creadora. [Internet]. 2016 [Consultado 19 septiembre de 2017]; 2(2):14-31. Disponible en: http:// revistas.udistrital.edu.co/ojs/index.php/estart/article/view/11525/12612

13. Camargo Sanchez A, Niño CL, Sánchez L, Echeverri S, Gutiérrez DP, et al. Theory of Inpatient Circadian Care (TICC): A Proposal for a Middle- Range Theory. Open Nurs J. 2015; 9(1):1-9.

14. Eisler A, Eisler H. Psychological time in Japanese and Swedish males: A cross-cultural comparison. Proceedings of Fechner Day. 2009; 25:311-316.

15. McEwan $\mathrm{H}$, Egan $\mathrm{K}$. La narrativa en la enseñanza, el aprendizaje y la investigación. 1st ed. Argentina: Amorrortu; 2005.

16. Charon R. Narrative Medicine: Honoring the Stories of Illness. 1st ed. Oxford University Press; 2006.

17. Cury M. Intertextualidade: uma prática contraditória. Cadernos de Linguística e Teoria da Literatura 1982;8:117-128.

18. Camarero J. Intertextualidad. 1st ed. Rubí, Barcelona: Anthropos; 2008.

19. Carvalhal T. Intertextualidade: a migração de um conceito. Via Atlântica. 2006; (9):125.

20. Zani R. Intertextualidade: considerações em torno do dialogismo. Em questão 2007; 9(1). 
ISSN-PRINT

1794-9831

E-ISSN 2322-7028

Vol. 15 No. 1

Ene - Jun 2018

Cúcuta, Colombia
21. Lightman A. Sueños de Einstein. 1st ed. Barcelona: Tusquets; 1994.

22. Granados Villlamil J, Parada Mise JP, Cáceres Serrano YM. Funcionalidad de las relaciones intrafamiliares en los pacientes con cáncer. Rev. cienc. cuidad. 2014; 11(1):27-34.

23. Cicogna E, Nascimento LC, Lima R. Children and Adolescents with Cancer: experiences with Chemotherapy. Rev Lat Am Enfermagem. 2010; 18(5):864-872.

24. Jowsey T. Time and chronic illness: a narrative review. Qual Life Res. 2015; 25(5):1093-1102.

25. Regina Secoli S, Pezo Silva MC, Alves Rolim M, Machado AL. El cuidado de la persona con cáncer: Un abordaje psicosocial. Index de Enfermería. 2005; 14(51):34-39.

26. Al Gamal E, Long T. Anticipatory grieving among parents living with a child with cancer. J Adv Nurs. 2010; 66(9):1980-1990.

27. Lewis JA, Manne SL, DuHamel KN, Vickburg SMJ, Bovbjerg DH, Currie V, et al. Social support, intrusive thoughts, and quality of life in breast cancer survivors. J Behav Med. 2001; 24(3):231-245.

28. Crespo Fernández L, Rivera García ML. El poder de la resiliencia generado por el cáncer de mama en mujeres en Puerto Rico. Rev. Puertorriq. Psicol. 2012; 23:109-126.

29. Gorea A. Ticks per thought or thoughts per tick? A selective review of time perception with hints on future research. J Physiol Paris. 2011; 105(4):153-163.

30. Llantá Abreu MC, Pire Stuart T, Grau Abalo J, Vilaú Prieto L, Massip Pérez C, et al. Evaluación del sufrimiento en pacientes con quimioterapia del Instituto Cubano de Oncología y Radiobiología. Psicología y Salud. 2013; 18(2):149-154.

31. Krikorian A. Valoración del sufrimiento en pacientes con cáncer avanzado. Psicooncología. 2008; 5(2):257-264.

32. Oyanadel C, Buela Casal G. La percepción del tiempo: influencias en la salud física y mental. Universitas Psychologica. 2011; 10(1):149-161.

33. Toboso Martín M. Fenomenología del transcurso del tiempo. Diánoia. 2007; 52(59):27-42.

34. Fernández Castro J, Granero Pérez R, Barrantes Vidal N, Capdevila A. Estado de ánimo y sesgos en el recuerdo: Papel del afecto. Psicothema. 1997; 9(2):247-258.

35. Whittemore R, Dixon J. Chronic illness: the process of integration. J Clin Nurs. 2008; 17(7):177-87.

36. Charmaz K. Good days, bad days: The self in chronic illness and time. Rutgers University Press; New Brunswick: NJ: 1991. 